\title{
Knee Imaging Following Anterior Cruciate Ligament Reconstruction: The Surgeon's and Radiologist's Perspectives
}

\author{
Ramya Srinivasan, MD ${ }^{1,2}$ Jennifer Wan, MD ${ }^{1}$ Christina R. Allen, MD ${ }^{3} \quad$ Lynne S. Steinbach, MD ${ }^{1}$ \\ ${ }^{1}$ Department of Radiology and Biomedical Imaging, University of \\ Address for correspondence Ramya Srinivasan, MD, University of \\ California San Francisco, Department of Radiology and Biomedical \\ imaging, 4150 Clement Street, Radiology (114), San Francisco, CA \\ 2 Department of Musculoskeletal Imaging, San Francisco Veterans \\ Affairs Medical Center, San Francisco, California \\ 94121 (e-mail: ramya.srinivasan@ucsf.edu).
}

${ }^{3}$ Department of Orthopedic Surgery, University of California, San

Semin Musculoskelet Radiol 2018;22:386-397.

\begin{abstract}
Keywords

- ACL reconstruction

- $\mathrm{ACL}$ reconstruction complications

- $A C L$ reconstruction MRI

- ACL graft tear

Anterior cruciate ligament $(\mathrm{ACL})$ tears are common injuries that if left untreated can result in chronic instability, cartilage damage, meniscal tears, and ligamentous injuries, eventually leading to early osteoarthritis. ACL reconstruction surgeries are therefore increasingly being performed. Despite the fact that most patients achieve excellent postoperative results, patients can present with recurrent instability and pain. These patients often undergo imaging with radiographs, magnetic resonance imaging, and/ or computed tomography. An understanding of the imaging appearance of the normal $\mathrm{ACL}$ reconstruction and common causes of failure is therefore essential for the interpreting radiologist. This article reviews surgical techniques for $A C L$ reconstruction, highlighting recent technical advances, the normal imaging appearance after $\mathrm{ACL}$ reconstruction, etiologies for reconstruction failure, and the diagnosis of these with the aid of imaging.
\end{abstract}

Anterior cruciate ligament (ACL) tears are common sports injuries with $\sim 250,000$ cases annually in the United States. ${ }^{1,2}$ These injuries most commonly occur during sports that involve deceleration, twisting, cutting, and jumping movements such as basketball, skiing, soccer, and football. ${ }^{3}$ Chronic instability associated with untreated ACL tears can lead to secondary injuries including cartilage damage, meniscal tears, and other ligamentous injuries, eventually leading to early osteoarthritis. ${ }^{2}$ Because of these devastating outcomes as well as improvements in surgical techniques, the incidence of ACL reconstruction surgeries has been rising with $\sim 100,000$ cases performed annually in the United States..$^{3,4}$ Although most patients treated with ACL reconstruction achieve excellent results, up to $15 \%$ of patients experience persistent instability and pain. ${ }^{4-6}$ Radiographs, computed tomography (CT), and magnetic resonance imaging (MRI) are all modalities used to evaluate ACL reconstructions. An understanding of the imaging appearance of normal ACL reconstruction and common causes of failure is therefore essential for the interpreting radiologist. This article reviews surgical techniques for ACL reconstruction, highlighting recent technical advances, the normal imaging appearance after ACL reconstruction, etiologies for reconstruction failure, and the diagnosis of these with the aid of imaging.

\section{Surgical Techniques}

\section{Bone-Patellar Tendon-Bone Autografts}

For the past 2 decades, ACL reconstruction utilizing bonepatellar tendon-bone (BPTB) autografts has been the gold standard. ${ }^{5}$ When compared with hamstring autografts, ВРТВ autografts were reported to show faster graft incorporation and a lower incidence of graft rupture. ${ }^{7}$ A recent meta-analysis comparing BРTB with hamstring autografts showed that reconstructions with BPTB autografts were less likely to
Issue Theme Postoperative Knee Imaging; Guest Editors, Marc-André Weber, MD, MSc and Donna G. Blankenbaker, MD
Copyright (c) 2018 by Thieme Medical Publishers, Inc., 333 Seventh Avenue, New York, NY 10001, USA. Tel: +1(212) 584-4662.
DOI https://doi.org/ 10.1055/s-0038-1653953. ISSN 1089-7860. 


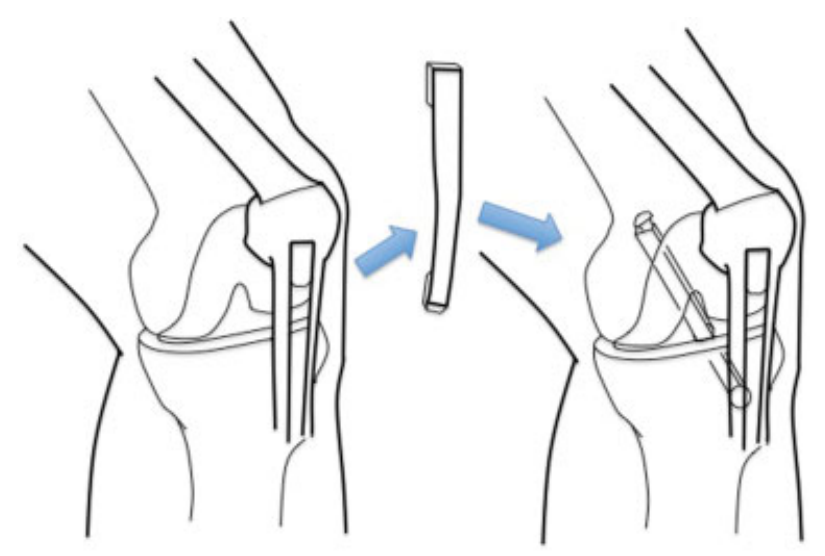

Fig. 1 Illustration shows harvesting and placement of the central patellar tendon with attached bone as the bone-patellar tendon-bone autograft.

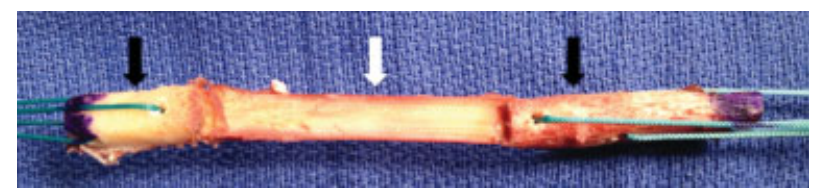

Fig. 2 Photograph of a harvested bone-patellar tendon-bone autograft. Note patellar tendon (white arrow) with bone plugs on at each end (black arrows).

undergo graft rupture or require revision surgery when compared with hamstring autografts (odds ratio: 0.83 ), although failure rates were low in both groups. ${ }^{8}$ The graft consists of the middle third of the patellar tendon along with plugs of bone harvested from the patellar and tibial attachments of the tendon ( - Figs. 1 and2). The graft is generally prepared to be 9 to $11 \mathrm{~mm}$ in diameter at the bone plug ends. ${ }^{2}$ The bone plugs on both ends are securely fixed in the femoral and tibial tunnels with either metal or bioabsorbable interference-fit screws that minimize the potential for graft loosening and loss of fixation. ${ }^{9}$

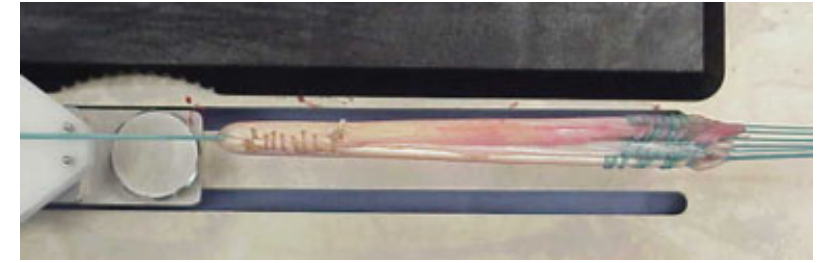

Fig. 4 Photograph of a harvested hamstring autograft. Note sutured doubled-over four-stranded graft.

\section{Hamstring Autografts}

Hamstring autografts have recently gained popularity due to increased postoperative pain as well as donor site and extensor mechanism complications related to BPTB autografts. ${ }^{10}$ The hamstring autograft typically consists of one hamstring tendon (semitendinosus) and one adductor tendon (gracilis) that are harvested from their tibial insertion up to the myotendinous junction of the individual tendons. The tendons are often sutured together and doubled over to create a fourstranded graft (-Figs. 3 and 4). The minimal acceptable graft size is optimally $7 \mathrm{~mm}$ in diameter (smaller grafts have a higher failure risk), and a graft length (when doubled over) of $\sim 9 \mathrm{~cm}$ is typically needed..$^{1-13}$ The femoral end of the graft is typically secured with a button or bioscrew, and the tibial end can be secured with staples, a washer and screw device, bioscrew, screw and sheath, or an extracortical button. ${ }^{10}$ A limitation of hamstring grafts is that the size of the graft depend on the size of the patient's hamstring tendons, and grafts $<7 \mathrm{~mm}$ in diameter were associated with increased laxity. ${ }^{12}$ This is in contrast to BPTB autografts, where the surgeon can determine the graft diameter during bone plug preparation. Another disadvantage of utilizing hamstring autograft is residual hamstring weakness that may hamper patient sprinting speed. ${ }^{14}$ In addition, the dynamic restraint capacity of the hamstring muscles is considered protective against ACL reinjury. ${ }^{15}$ Potential hamstring weakness after graft harvesting may therefore increase the risk of ACL reinjury when the patient returns to sports.

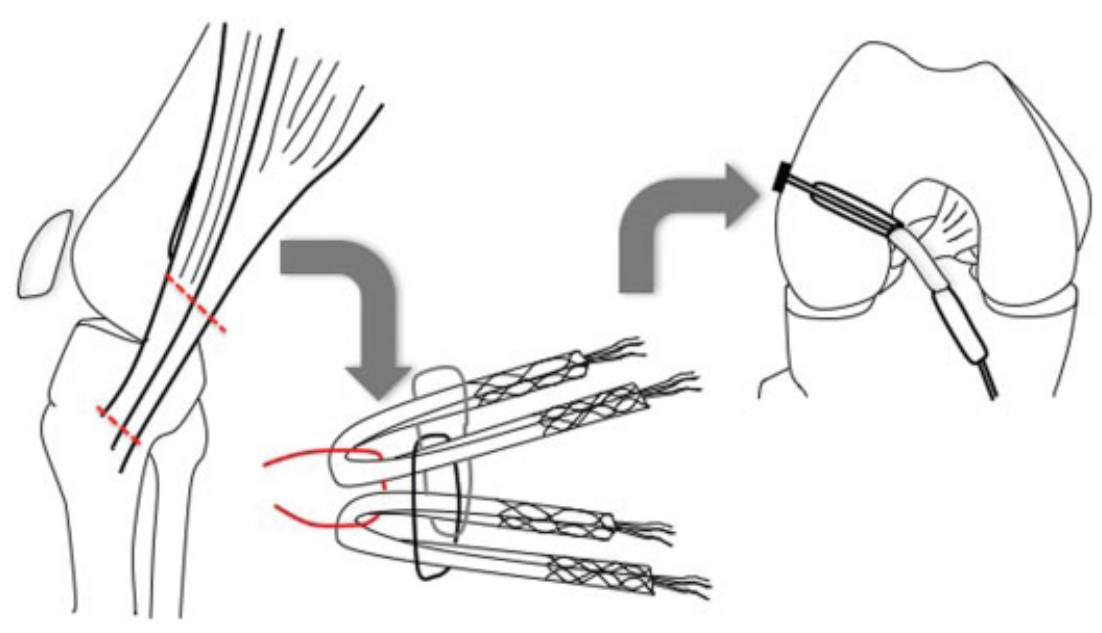

Fig. 3 Illustration shows harvesting of the semitendinosus and gracilis tendons from their musculotendinous junctions to their tibial insertion, suturing of the tendons into a four-stranded graft, and placement of the autograft fixed with an EndoButton at the femoral cortex. 


\section{Allografts}

Allografts are tendons harvested from cadavers. Commonly used allografts include BPTB, Achilles, hamstring, quadriceps, posterior tibialis, anterior tibialis, and peroneus longus tendons. ${ }^{16}$ Advantages of allografts include elimination of donor site morbidity, decreased surgery time, smaller skin incisions, and a decreased incidence of arthrofibrosis. ${ }^{13}$ However, several risks are specific to allografts. Many reports demonstrated slower speed of graft incorporation and "ligamentization" and therefore a higher failure rate for allografts, especially in younger patients. ${ }^{17}$ An additional concern is the risk of transmission of viral and bacterial infections, although the overall risk is low, $\sim 0.14$ to $0.17 \%{ }^{18} \mathrm{~A}$ higher incidence of bone tunnel enlargement is also a potential risk. ${ }^{13}$ Additional disadvantages include alteration of mechanical properties of the graft due to sterilization and preparation of the graft. Allografts are therefore typically reserved for cases of multiligament injuries and revision ACL reconstructions when there is no remaining autograft source, or in older patients who will return to sports more slowly and/or participate in activities that place a lower demand on the ACL graft, therefore lowering the risk of ACL graft failure.

\section{Synthetic Grafts}

In the 1980s, synthetic grafts became an attractive alternative to biological grafts because of the lack of donor site morbidity associated with autografts, the strength of the synthetic graft and ability for immediate loading, and reduced postoperative rehabilitation time. ${ }^{19}$ Synthetic materials used include carbon fibers, polypropylene, Dacron, and polyester. All of these materials can lead to potential complications including infection, host immunologic responses, breakage, synthetic debris leading to synovitis, chronic effusions, recurrent instability, and osteoarthritis. Because of these complications, the use of synthetic grafts has been limited. However, recent studies found that under specific conditions, synthetic grafts can be successful. ${ }^{20,21}$ This has led to a recent renewed interest in the use of synthetic grafts. Research into finding the ideal synthetic substitute with regard to biocompatibility and mechanical characteristics is still ongoing.

\section{Biological Options}

Recent basic science research has suggested that combining suture repair techniques for a torn ACL with a biological adjunct may improve the result of primary ACL repair. ${ }^{22}$ Various growth factors have been studied that were shown to stimulate collagen production; however, delivery of these growth factors to the ACL tear site in a sustained fashion has been a challenge. Autologous platelets contain multiple growth factors and are capable of forming a clot and can be delivered in the form of platelet-rich plasma (PRP) or whole blood. However, the use of PRP had mixed results in porcine models. ${ }^{23}$ This may be because the main protein in the clotted PRP is fibrin, which is degraded by the plasmin present in synovial fluid. This has led to the exploration of carriers to hold the PRP at the wound site and to protect it from early degradation. A collagen-PRP composite was shown in porcine models to improve the biomechanical properties of the repaired ACL when compared with suture repair alone. ${ }^{24}$ Although bio-enhanced ACL repair showed promise in animal models, clinical data are not yet available, although the technique was recently approved by the U.S. Food and Drug Administration for a human clinical safety study.

Another potential biological option is stem cell therapy in conjunction with ACL reconstruction. This showed some promise in animal models, but clinical data are limited. ${ }^{25}$ Silva et al compared patients who underwent ACL reconstruction with and without grafts injected with adult noncultivated bone marrow stem cells. ${ }^{26}$ These patients underwent MRI to evaluate for tendon to bone healing as evidenced by changes in signal on proton-density (PD) and $\mathrm{T} 1$-weighted sequences. The conclusion was that stem cells do not seem to accelerate graft tendon to bone healing in ACL reconstruction.

\section{Double-Bundle and Selective-Bundle Reconstruction}

The native ACL is composed of two bundles that act synergistically with the anteromedial bundle taut with flexion and the posterolateral bundle taut with extension. ${ }^{27}$ Both bundles are restraints to anterior tibial translation, and the posterolateral bundle is largely responsible for providing rotational stability. Single-bundle reconstructions (SBRs) most closely replicate the orientation of the anteromedial bundle, especially when the older transtibial ACL reconstruction technique is utilized. Newer two-incision and anteromedial femoral tunnel drilling techniques are designed to be more isometric and center the ACL femoral tunnel between the anteromedial and posterolateral bundle insertions on the femur. ${ }^{28,29}$

Because of residual instability and patient dissatisfaction in a subset of patients after SBR, double-bundle reconstructions (DBRs) have emerged with the aim to recreate normal knee kinematics more closely and provide greater knee stability. There have been mixed results. Some studies comparing SBR with DBR showed no significant differences in measures of knee stability, knee scores, subjective evaluation, and MRI evaluation of graft inclination. ${ }^{30,31}$ The process of DBR involves drilling two tunnels into the tibia and two into the femur. Anterior tibialis tendon or hamstring allografts are typically used with occasional use of hamstring autografts. ${ }^{32}$ The femoral ends of the grafts are secured with button fixation, and the tibial ends are secured with interference screws with or without staple reinforcement.

Selective-bundle reconstruction aims to repair the injured anteromedial or posterolateral bundle while preserving the remaining intact bundle. The injured bundle is usually reconstructed with a soft tissue graft such as a hamstring autograft or allograft. ${ }^{33}$

\section{Imaging Evaluation of ACL Reconstruction}

\section{The Normal ACL Graft}

Immediately following ACL reconstruction surgery, the ACL graft appears as a homogeneous thick band of hypointense signal on T1- and PD-weighted images. At 4 to 8 months after reconstruction, the process of ligamentization starts with synovial proliferation and vascularization of the graft tendon. ${ }^{34}$ This leads to increased signal on all MRI sequences that 
decreases with time. The literature varies regarding how long the process of ligamentization takes. Some authors report that the process is completed by 1 year after reconstruction. Other authors report that the process continues for 2 years. ${ }^{34-36}$ A study by Saupe et al, however, demonstrated that small amounts of increased intrasubstance signal can be seen $\geq 4$ years after reconstruction, and this does not correlate with joint instability or functional limitations. ${ }^{31,37}$ Importantly, because of its four-stranded construction, the hamstring tendon autograft can initially demonstrate intermediate signal or fluid between the strands of the graft on T2-weighted sequences. The intermediate or high signal is oriented parallel to the fibers of the graft as opposed to a tear that would demonstrate signal abnormality perpendicular to the graft. ${ }^{9}$ This normal finding in the hamstring graft would be abnormal in a BPTB autograft. It was also reported that the ligamentization process in allografts is less uniform and more prolonged than in autografts. ${ }^{38}$

\section{The Femoral and Tibial Tunnels}

Correct tunnel positioning is crucial for maintaining graft integrity and stability. Malpositioning of the tunnels is one of the most common technical errors in ACL reconstruction, and it is estimated that up to $80 \%$ of graft failures are due to improper tunnel placement. ${ }^{39,40}$

The position of the femoral tunnel determines graft isometry, the constancy in length and tension of the graft during the complete range of knee motion. ${ }^{36,41}$ In the past, surgical techniques for ACL reconstruction (especially transtibial ACL reconstructions) would orient the femoral tunnel in the sagittal plane so the inferior end of the femoral tunnel was located at the intersection of a line along the posterior cortex of the distal femoral metadiaphysis and a line along the roof of the intercondylar notch (Blumensaat's line). ${ }^{36}$ The clock method was also used for orienting the femoral tunnel in the coronal plane with the tunnel placed at the 10 to 11 o'clock position in the right knee and the 1 to 2 o'clock position in the left knee. ${ }^{41}$ These methods aimed to mimic the femoral insertion site of the anteromedial bundle of the native ACL.

Modern ACL reconstructions now commonly use the quadrant method to localize and position the femoral tunnel that more closely replicates the native femoral ACL insertion. ${ }^{42}$ With the knee flexed to 90 degrees, a true lateral radiograph is obtained. An imaginary box can be drawn with the superior border drawn along Blumensaat's line, the inferior border drawn at the inferior border of the femoral condyles, and the anterior and posterior borders at the anterior and posterior margins of the femoral condyles. This box can then be divided into a grid by quartering the sagittal diameter and the notch height. Optimal placement of the femoral tunnel aperture is at the anteroinferior corner of the superoposterior quadrant (-Fig. 5). ${ }^{39,42,43}$

The position of the tibial tunnel is crucial in preventing impingement of the ACL graft against the roof of the intercondylar notch in the sagittal plane or the posterior cruciate ligament or lateral femoral condyle in the coronal plane. ${ }^{29} \mathrm{On}$ sagittal images, the tunnel should be oriented parallel to Blumensaat's line when the knee is in full extension, and the

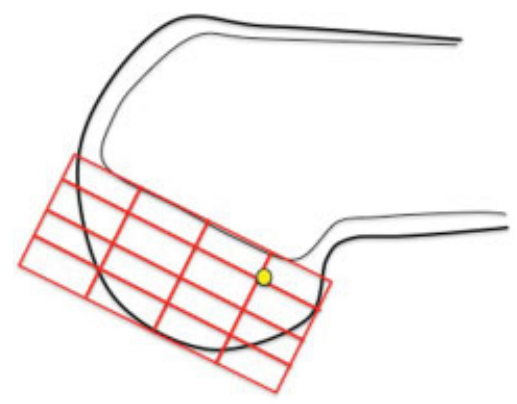

Fig. 5 Illustration of the quadrant method with the aperture of the femoral tunnel at the anteroinferior corner of the superoposterior quadrant (yellow circle).

intra-articular opening should be posterior to where Blumensaat's line intersects the tibia when the knee is in full extension. The Staubli and Rauschning technique is often used for tibial tunnel placement. Using this method, the center of the aperture of the tibial tunnel is located $43 \%$ along the Amis and Jakob line ${ }^{43}$ (-Fig. 6). The Amis and Jakob line is a horizontal line drawn from anterior to posterior in the sagittal plane through the medial tibial plateau. In the coronal plane, the distal end of the tunnel should start medial to the tibial tuberosity, and the proximal end should enter the joint between the two tibial intercondylar spines ${ }^{36,41}$ and be oriented at 65 to 70 degrees to a horizontal line drawn along the tibial plateaus.

Rotational instability (clinically evidenced by a positive pivot shift) is associated with femoral tunnels that are placed too anteriorly and/or femoral or tibial tunnels placed too vertically in the coronal plane. ${ }^{39}$

Tunnel position, width, and shape should be assessed in both the coronal and sagittal planes on follow-up radiographs, CT, and/or MR images. The walls of the tunnel should

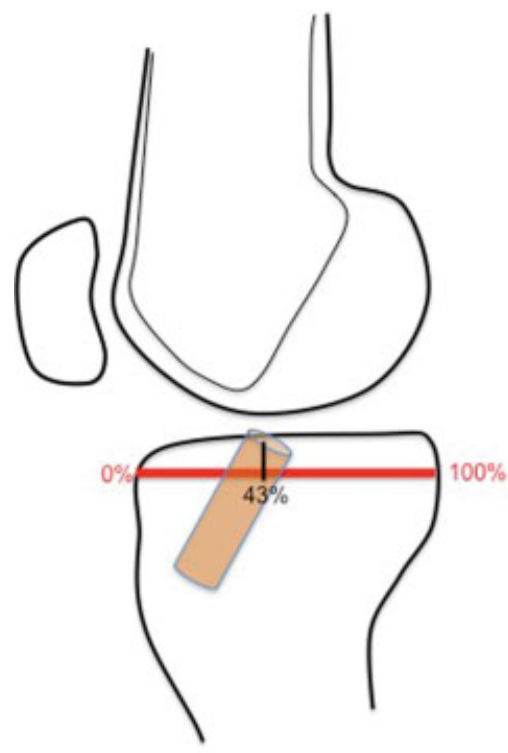

Fig. 6 Illustration of tibial tunnel positioning in the sagittal plane using the Staubli and Rauschning technique with the center of the tibial tunnel located $43 \%$ along the Amis and Jakob line. 
be parallel, with a width $\sim 10 \mathrm{~mm}$; however, an upper limit of normal of $13 \mathrm{~mm}$ was reported during the first 18 months after surgery. ${ }^{34,39,44}$ Early postoperative radiographs serve as a baseline for future reference.

Although tunnels are most often evaluated postoperatively on radiographs, some authors have endorsed the use of CT with sagittal and coronal reformats and three-dimensional volume rendered images as a more accurate and reliable method for evaluating tunnel placement and morphology as compared with standard radiographs. ${ }^{39,45}$ Early postoperative $\mathrm{CT}$ is increasingly being used to provide immediate feedback on tunnel positioning as well as fixation and device placement.

\section{Normal MRI Appearance of the Harvest Sites}

In the case of BPTB autograft reconstructions, MRI shows a thickened patellar tendon with a defect in the central patellar tendon, unless the surgeon closed the defect with sutures. This appearance can be present until 2 years after surgery after which the patellar tendon resumes its normal appearance. A defect at the donor site of the patella can be seen up to a year postoperatively. ${ }^{9,36}$ The tibial bone harvest donor site defect may persist even longer.

MRI of the hamstring harvest site initially reveals absence of the tendons with a fluid-filled defect. The tendons were reported to regenerate over a 2.5- to 3-year period in a proximal to distal fashion. ${ }^{46}$

\section{ACL Reconstruction Complications}

Although there have been significant technical improvements in ACL reconstruction techniques in the past 15 years, the rate of graft failure is substantive. A systematic review conducted by Reinhardt et al found that failure rates ranged from $7.2 \%$ to $15.8 \%{ }^{5}$ A meta-analysis performed by Crawford et al found that at $>10$-year follow-up, ACL graft rupture rate was $6.2 \%$ and clinical failure occurred in $\sim 10.3 \%{ }^{6}$

Complications related to the graft itself can be roughly divided into those that result in increased laxity and those that result in decreased range of motion (flexion, extension, or both).

Causes of laxity include partial or complete tears of the graft, graft stretching/elongation, graft malplacement, and fixation failure. Causes of decreased range of motion include roof impingement, arthrofibrosis and cyclops lesions, osteophyte formation, intra-articular bodies, and mucoid degeneration of the graft with associated ganglia.

Additional complications include tunnel widening, hardware complications, harvest site complications, extensor mechanism dysfunction, and osteoarthritis.

\section{Complications Leading to Laxity}

\section{Graft Tears}

Grafts are most susceptible to injury during the ligamentization period, particularly in the first 8 months after ACL reconstruction. ${ }^{36}$ Tears can be the result of surgical error, failure in graft incorporation, untreated injuries to secondary restraints, repeat trauma, infection, or a combination of these factors. ${ }^{4}$ If the reconstruction fails within 6 months after surgery, a technical component usually plays a role. ${ }^{47}$ Among surgical technical errors, malpositioned tunnels are the most common, leading to excess stress on the graft that can result in a tear or stretching/elongation. Other surgical errors include inadequate fixation and screw divergence between a screw and a graft that contains bone plugs. The optimal orientation of the screw within the tunnel for maximum fixation strength is parallel to the graft. Divergence beyond 30 degrees is abnormal and can compromise fixation strength leading to graft failure. ${ }^{39}$

On imaging, primary and secondary signs of complete graft tears can be seen. Primary signs on MRI include discontinuity of graft fibers, graft thinning, increased graft signal, and lax graft fibers or horizontal orientation ${ }^{9,45}$ (-Fig. 7). It is important to recognize that tears can occur not only in the intra-articular portion of the graft but also within the tibial or femoral tunnels. Graft fiber continuity should therefore be carefully assessed in the tunnels. Secondary signs of graft tears include anterior tibial translation, uncovering of the posterior horn of the lateral meniscus, and buckling of the posterior cruciate ligament. ${ }^{36}$

In a study by Horton et al evaluating ACL graft MRI findings (using arthroscopy as the gold standard for determining ACL graft integrity), ACL graft fiber continuity in the coronal plane and $100 \%$ graft thickness in the sagittal or coronal plane were the most predictive findings for excluding full-thickness graft tears. ${ }^{45}$ Complete discontinuity of the graft in the coronal plane was valuable for diagnosing a fullthickness tear. Secondary signs were found not to be particularly useful largely due to low sensitivities. In addition, many of the secondary signs were present with intact grafts. The authors postulated that these results might indicate the intact grafts did not provide the same degree of stability as

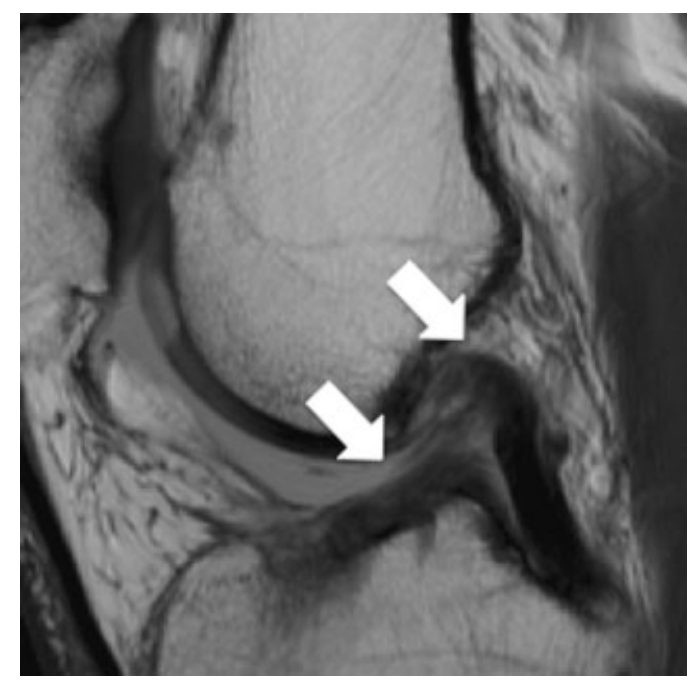

Fig. 7 Sagittal proton-density-weighted MR image of a 35-year-old man who presented with knee pain and swelling after trauma with a Lachman-1A score on examination demonstrates a complete tear of the $A C L$ graft with lack of visualization of graft fibers in the intercondylar notch (arrows). 
the native ACL. Another explanation was inconsistency in identification of secondary signs, which showed only fair to moderate interobserver agreement. ${ }^{45}$

The same authors also found relative insensitivity in the detection of partial ACL graft tears on MRI. A possible explanation is that the surgeon takes into consideration graft tautness in addition to anatomical appearance when diagnosing partial tears. A graft that is lax at arthroscopy may appear morphologically normal on MRI. Fortunately, partial tears are usually treated conservatively, and the important distinction to make is between a full-thickness tear and other conditions (intact graft or partial-thickness tear).

\section{Graft Stretching}

Graft stretching or elongation can contribute to knee laxity or instability (positive Lachman's examination or pivot shift) with an intact graft. ${ }^{36,48}$ Graft stretching can result from malpositioned femoral and tibial tunnels. A femoral tunnel placed too far anteriorly subjects the graft to increased strain when the knee is flexed (as well as graft impingement in extension) that may lead to graft stretching. It was also suggested that there is a higher incidence of stretching with hamstring tendon autografts. ${ }^{36,48}$

On MRI, the stretched graft demonstrates posterior bowing. In addition, the femoral tunnels may be widened from backand-forth motion of the graft with flexion and extension. There may also be anterior translation of the tibia secondary to anterior laxity. ${ }^{46,49}$

Anterior laxity may also result from postoperative thickening of the posterior capsule causing anterior translation of the tibia. ${ }^{34}$

\section{Complications Leading to Decreased Range of Motion}

\section{Arthrofibrosis and Cyclops Lesions}

The cyclops lesion is an ovoid lesion located in the intercondylar notch, connected to the ACL graft and usually located anteriorly. The cyclops lesion owes its name to the arthroscopic appearance of the ovoid soft tissue mass with venous vessels resembling an eye. ${ }^{48}$ The origin of the lesion is uncertain, but proposed etiologies include reaction to the debris from drilling of the tibial tunnel, reaction to the torn ACL stump, and microtrauma to the graft leading to an inflammatory process and formation of the cyclops lesion. ${ }^{1,50}$ Removing debris from drilling of the tibial tunnel and thorough debridement of the ACL stump can help prevent the formation of a cyclops lesion. ${ }^{4}$ Two distinct histologic subtypes were described. ${ }^{51}$ The true cyclops lesion is a hard lesion composed of fibrocartilaginous tissue with active bone formation in the center. A similar but soft lesion has been referred to as a "cyclopoid" lesion composed of fibrocartilaginous islands surrounded by granulation tissue. Nevertheless, both of these subtypes are commonly referred to as cyclops lesions. On MRI, these lesions appear as well-circumscribed nodules demonstrating hypointense to intermediate signal intensity on T1-weighted images and variable signal intensity on PD and T2-weighted images (-Fig. 8). The differential diagnosis includes nodular pigmented villonodular synovitis and synovial chondromatosis. ${ }^{34,41}$

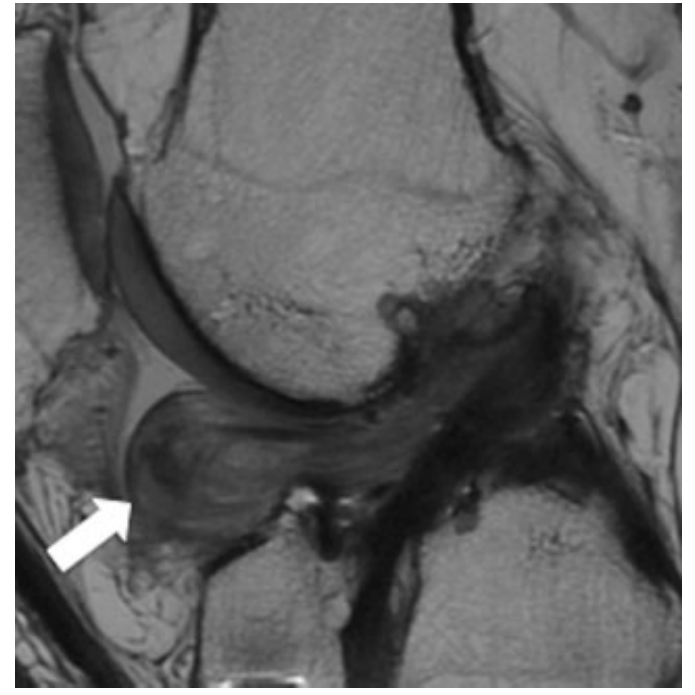

Fig. 8 Sagittal proton-density-weighted MR image of a 33-year-old woman who presented with pain demonstrates a large heterogeneous signal lesion in the intercondylar notch compatible with a cyclops lesion (arrow).

Fibers from a partial tear of the ACL graft can produce an appearance similar to a cyclops lesion, termed the "pseudocyclops lesion"52 (- Fig. 9).

Previous reports stated that cyclops lesions occur in 1 to $10 \%$ of cases after ACL reconstruction and are a common cause of loss of terminal knee extension. ${ }^{1,34}$ In these studies, the prevalence of cyclops lesions was assessed only in subjects undergoing arthroscopy for severe loss of knee extension. A recent prospective study by Facchetti et al, following 113 patients who underwent ACL reconstruction, found that cyclops lesions are detected on MRI with a prevalence of $25 \%$ at 6 months after surgery, and subjects with cyclops lesions did not have an inferior clinical outcome compared with

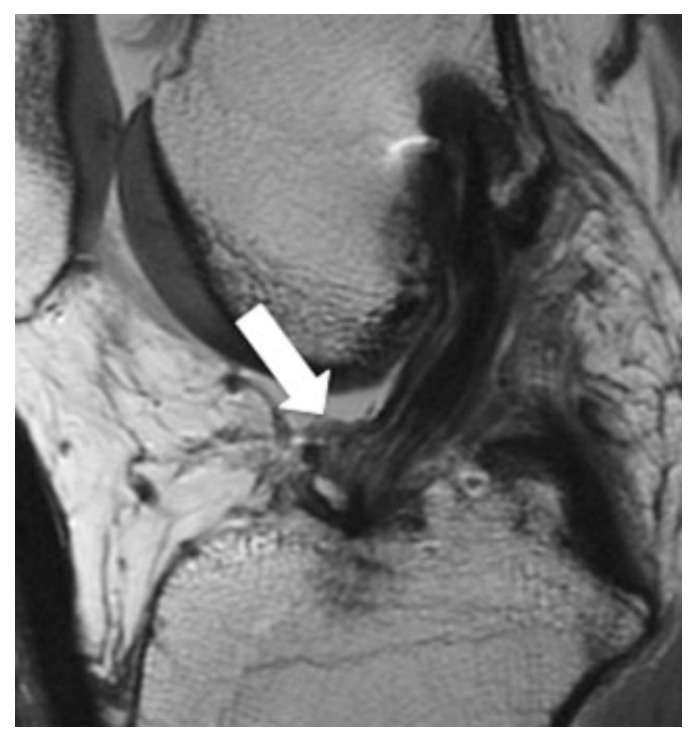

Fig. 9 Sagittal proton-density-weighted MR image of a 50-year-old man presenting to rule out medial meniscal tear demonstrates a partial tear of the graft involving the anterior/distal fibers that appear redundant and displaced anteriorly (arrow), resulting in a pseudocyclops lesion. 


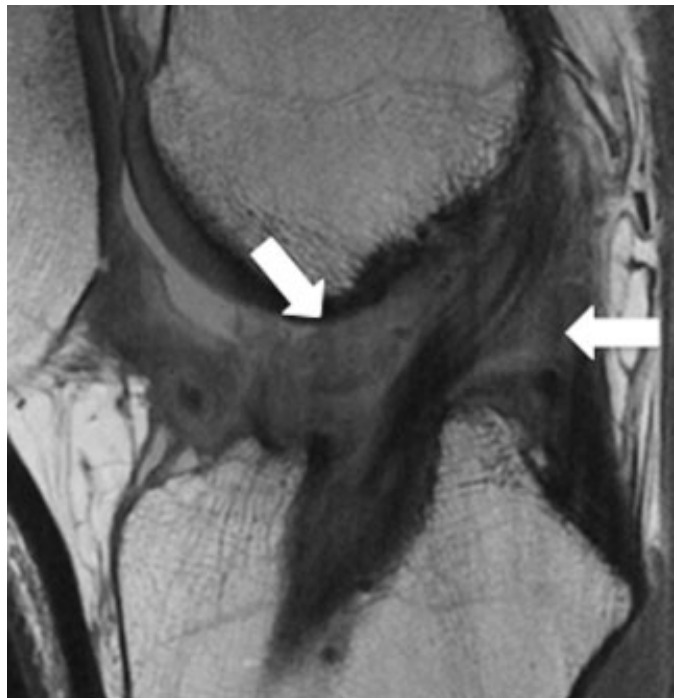

Fig. 10 Sagittal proton-density-weighted MR image of a 47-year-old man who presented with anterior knee pain and difficulty with step-up demonstrates amorphous intermediate signal material anterior and posterior to the graft (arrows), compatible with arthrofibrosis.

those without. ${ }^{1}$ They concluded that the reason why only some cyclops lesions can cause loss of extension needs further investigation.

Symptomatic cyclops lesions that block full extension, also referred to as "cyclops syndrome," are treated with arthroscopic debridement. ${ }^{1}$

The diffuse form of postoperative arthrofibrosis occurs less commonly than cyclops lesions and on MRI appears as spiculated, sometimes mass-like areas of hypointense signal located in the anterior and/or posterior compartments and is often seen with capsular thickening ${ }^{36}$ (-Fig. 10). It is the result of inflammation that results in adhesions and scar formation. ${ }^{4}$ The increased inflammation may be the result of an abnormal patient response to surgery or trauma or may be secondary to surgery performed too soon after ACL injury, poor surgical technique, or restricted postoperative motion. ${ }^{4,41}$ Patients present with decreased range of motion with both flexion and extension. Treatment depends on the stage of arthrofibrosis. In the early inflammatory stage, treatment should focus on the reduction of pain and inflammation. In the later fibrotic stage, arthroscopic debridement is typically performed. ${ }^{53}$

\section{Graft Impingement}

Tibial tunnel malposition is the primary factor leading to ACL graft impingement. If the tibial tunnel is positioned too far anteriorly, the graft can be impinged on by the roof of the intercondylar notch during terminal knee extension. ${ }^{36}$ Impingement may also occur secondary to anterior translation of the tibia that moves the tibial tunnel anteriorly, as can be seen in the setting of anterior laxity. ${ }^{36,41}$ On MRI sagittal views, the graft is posteriorly bowed due to impingement against the top of the notch and may show signal alteration in the anterior two thirds of the graft. ${ }^{34,41}$ Less commonly, the tibial tunnel is placed too far laterally and the graft impinges on the lateral sidewall of the intercondylar notch. If the tunnel is placed too medially and vertically, the graft can impinge on the posterior cruciate ligament. ${ }^{34}$

Inadequate notchplasty can also lead to impingement, although with current ACL reconstruction techniques that orient the graft less vertically, notchplasty is less commonly needed. ${ }^{54}$ Osteophytes and scarring can also lead to impingement. Patients with impingement present with a block to full knee extension and morning stiffness that improves as the day progresses. ${ }^{4}$ Early treatment involves serial extension casting or the use of an extension dropout cast. ${ }^{41}$ Chronic cases may require revision notchplasty and debridement of the scar tissue. Occasionally, revision ACL reconstruction to revise tibial tunnel position is required to regain extension.

\section{Mucoid Degeneration of the Graft}

Mucoid degeneration of the graft with graft ganglia occurs as a late complication or incidental finding. On MRI there is expansion of the graft with cystic lesions interdigitating between intact graft fibers ${ }^{34,44}$ (-Fig. 11). Cystic lesions can also be seen adjacent to the graft within the tibial tunnel with resulting expansion of the tunnel and surrounding reactive marrow edema (-Fig. 12). Mucoid degeneration of the graft is not associated with graft failure, but ganglion cysts may cause pain and, if large enough, restricted range of motion. ${ }^{9,36}$

\section{Intra-articular Bodies}

Intra-articular bodies can be present after ACL reconstruction and may cause decreased range of motion. These bodies may result from chondral injuries that occurred at the time of initial ACL injury that were not seen during ACL reconstruction. $^{36,48}$ The bodies may be composed of articular cartilage, cortical bone, and/or cancellous bone, therefore resulting in variable signal intensity on MRI ranging from intermediate to

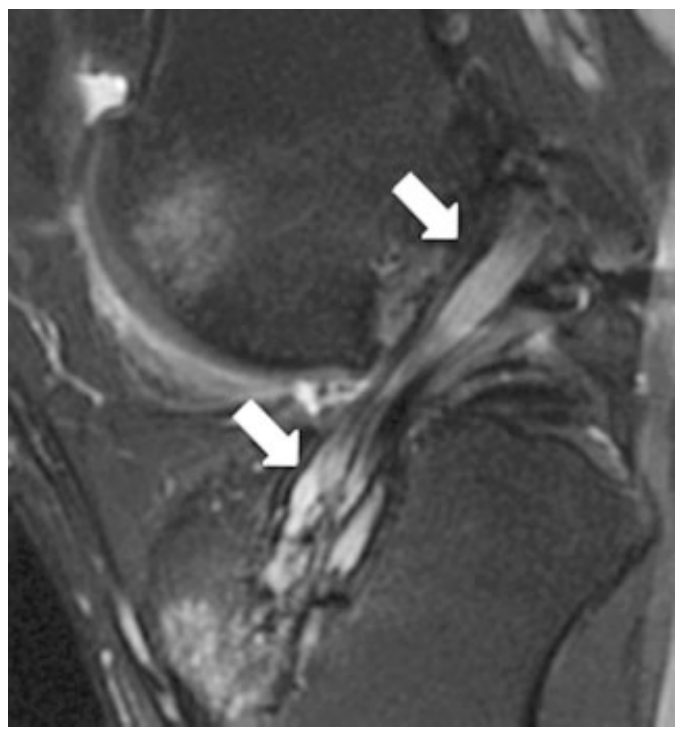

Fig. 11 Sagittal T2-weighted fat-suppressed image of a 42-year-old man who presented with anterior tibial pain demonstrates expansion of the $\mathrm{ACL}$ graft with $\mathrm{T} 2$ hyperintense lesions interdigitating with the graft fibers both in the intercondylar notch and in the tibial tunnel (solid white arrows), compatible with mucoid degeneration. 


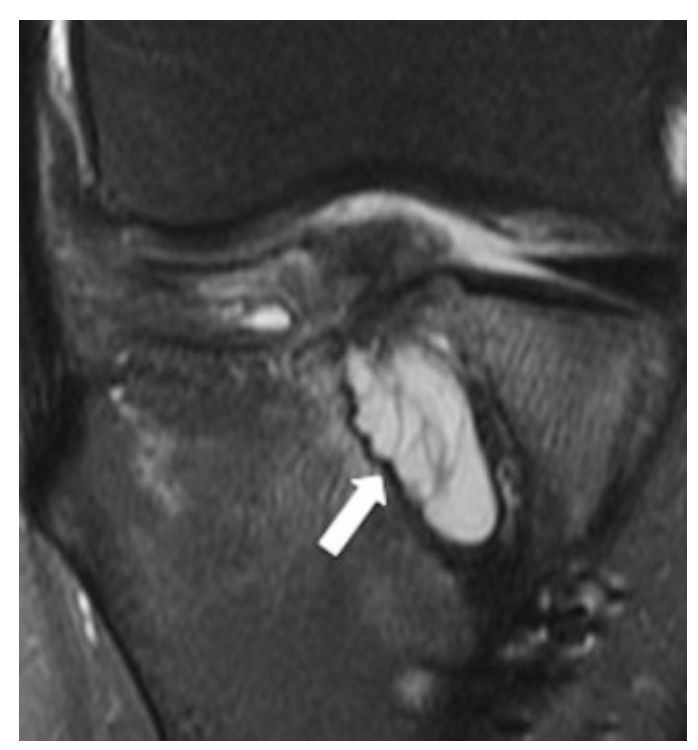

Fig. 12 Coronal T2-weighted fat-suppressed image of a 29-year-old woman who presented with pain and lack of full range of motion in extension demonstrates cystic lesions expanding the tibial tunnel (arrow) with surrounding marrow edema.

hypointense on T2-weighted sequences. MR arthrography can be helpful by increasing the sensitivity for detection of these bodies. $^{36}$

Bone shavings created during tunnel drilling and/or notchplasty can accumulate due to inadequate evacuation of bone debris and appear as small intra-articular bodies. In a study by Seeger et al, small linear intra-articular calcifications, usually in the posterior knee joint, were commonly seen on initial postoperative radiographs. In those patients who obtained follow-up radiographs within 6 years, the calcifications had resolved. ${ }^{55}$ The authors, however, could not determine the time frame for their disappearance. They concluded that these calcifications were of doubtful clinical significance. They also suggested that an alternative source of these calcifications might be secondary chondrocalcinosis, reported to occur with posttraumatic etiologies such as osteochondritis dissecans or after meniscectomy.

\section{Miscellaneous Complications}

\section{Tunnel Enlargement}

Tunnel widening affects the tibial tunnel more commonly than the femoral tunnel and tends to occur in the first 6 months, stabilizing within 2 years. ${ }^{56}$ No correlation was found between tunnel widening and graft failure; however, tunnel widening may cause problems if revision surgery is needed. Although tunnel widening is a frequent phenomenon, little is known regarding its cause or natural history. Several biological and mechanical etiologies for tunnel enlargement were implicated including nonanatomical graft fixation and improper tunnel placement, bioscrew resorption and reaction, ACL graft ganglia, foreign body immune response to allografts, heat necrosis from drilling, cytokine-mediated inflammatory responses, and cell necrosis due to toxic ethylene oxide and metal. ${ }^{44}$ In addition, early aggressive rehabilitation may contribute to tunnel enlargement because of stresses placed at the graftbone interface before incorporation and ligamentization is complete.

Tunnel enlargement is seen more commonly with hamstring autografts versus BPTB autografts, potentially because of the use of suspensory fixation devices and lack of a bone on bone interface, leading to increased micro-motion. ${ }^{13}$ It was also reported that tunnel expansion occurs more often when bioabsorbable rather than metal screws are used for graft fixation. ${ }^{44}$ Younger age, male sex, and delay from the time of injury to ACL reconstruction are additional potential risks for enlargement. $^{57}$

Tunnel cyst formation is a rare complication after ACL reconstruction that can result in tunnel expansion. These cysts also occur more commonly in the tibial tunnel than in the femoral tunnel. ${ }^{34}$ Patients may present with pretibial swelling or a palpable mass with or without pain if the cysts expand into the anterior soft tissues over the distal tibial tunnel. $^{36,56}$ The pathogenesis of these cysts is unclear. As mentioned previously, these cysts may result from cystic degeneration of the ACL. Other proposed causes include necrosis, foreign body reaction to the screw material (especially bioabsorbable screws), lack of complete graft osteointegration, and intravasation of joint fluid. ${ }^{56}$

It is important to note that a small amount of fluid within the tunnels can be a normal postoperative finding on MRI that typically resolves within 18 months. ${ }^{56}$

In the case of ACL graft failure and revision reconstruction, tunnel diameter is a factor in surgical planning. If the tibial or femoral tunnel diameter exceeds $15 \mathrm{~mm}$, a two-stage procedure is needed with initial bone grafting to fill the tunnels followed at least 3 months later by revision surgery. ${ }^{44}$ Revision with tunnel diameters $<10 \mathrm{~mm}$ can usually be performed in a single stage, depending on graft options available. For tunnel sizes between 10 and $15 \mathrm{~mm}$, the need for bone grafting depends on the shape of the tunnel and anticipated graft choice. The use of BРTВ grafts in revision surgery can allow single-stage revisions to be performed in tunnel diameters up to $12 \mathrm{~mm} .{ }^{44}$

\section{Fixation Site Complications}

The stability of the initial fixation is crucial for the success of ACL reconstruction. ${ }^{4}$ Secure fixation is important in preventing changes in graft position during the initial 6- to 12-week postoperative period during which graft-tunnel incorporation takes place. Complications related to fixation include hardware fracture and migration, bone plug fracture, or failure of bone plug incorporation. ${ }^{9}$ Although interference screws are considered the best method of fixation for grafts containing bone plugs, their use is associated with several potential complications. Interference screws can migrate into the intercondylar notch, which may lead to graft impingement or cartilage damage ( $\mathbf{F i g . ~ 1 3 a , ~ b ) . ~ S c r e w s ~ m a y ~ a l s o ~}$ migrate to an extra-articular location or diverge from the bone plug resulting in loss of graft fixation and therefore ACL graft failure. ${ }^{34}$ If the tibial screw migrates anteriorly out of the distal tibial tunnel, the patient can present with a palpable lump and pain (-Fig. 14). Metallic interference 


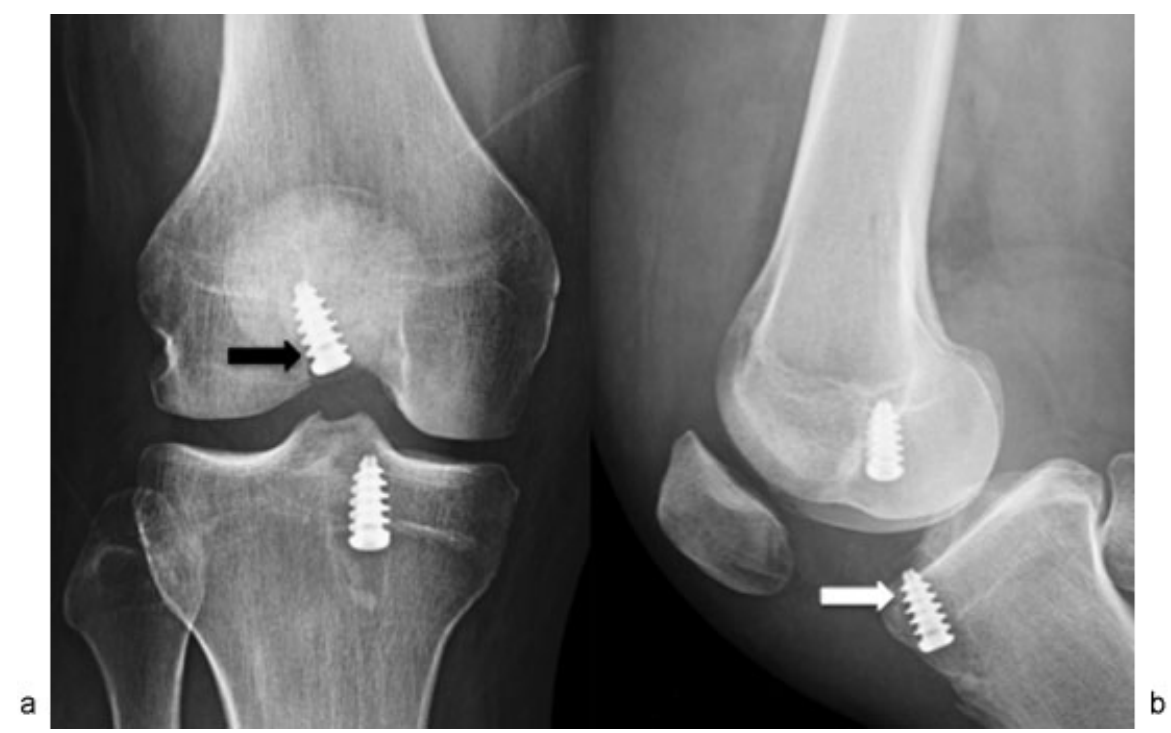

Fig. 13 (a) Anteroposterior radiograph of a 31-year-old man who presented with knee pain demonstrates the femoral interference screw projecting into the intercondylar notch (arrow). (b) Lateral radiograph in the same patient demonstrates a mildly proud tip of the tibial interference screw projecting into the intercondylar notch (arrow).

screws carry the added concerns of distortion on postoperative MRI evaluation and the requirement for removal during revision surgery. ${ }^{58,59}$

Bioabsorbable screws have been used for graft fixation to avoid concerns related to metal screws. Commonly used materials include polyglycolic acid and polyparadioxanone among several other materials. These implants are designed to degrade over time. Concerns specific to bioabsorbable screws include intraoperative screw breakage, an inflammatory foreign body reaction, incomplete absorption, increased cost, and as previously mentioned, bone tunnel widening. Interestingly, a meta-analysis by Emond et al found no significant difference

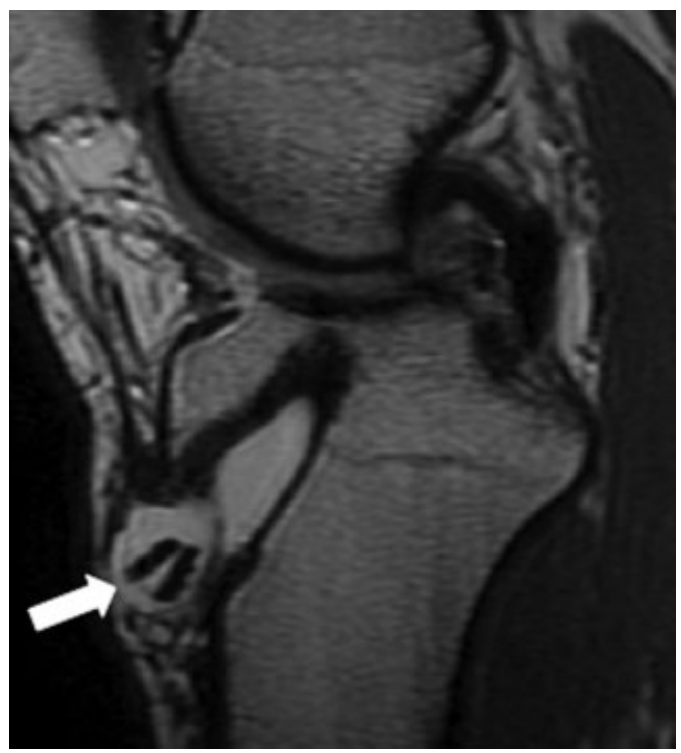

Fig. 14 Sagittal proton-density-weighted MR image of a 19-year-old man who presented with a palpable lump and pain at the tibial screw site demonstrates anterior extrusion of a fragmented tibial screw into the subcutaneous soft tissues (arrow). in outcomes associated with the use of bioabsorbable and metal screws, although they also concluded that further investigation into the differences in tunnel widening is needed. ${ }^{58}$ Bioabsorbable cross pins have also been used for femoral fixation of the graft and can fracture or migrate. However, in a study by Studler et al, these findings did not correlate with joint instability or pain. ${ }^{60}$

Cortical button positioning (such as the EndoButton or RetroButton) can be evaluated with radiography and should normally be seated on the femoral cortex. ${ }^{34,39}$ Tissue interposition, likely representing periosteum or in some case the iliotibial band, can result in a gap $>1 \mathrm{~mm}$ between the button and femoral cortex. This can be seen immediately postoperatively on radiographs, although this finding was not shown to affect long-term outcomes. ${ }^{61}$ Cortical buttons can also migrate into the femoral tunnel and knee joint due to lateral femoral cortex breakout not recognized intraoperatively, resulting in a complete loss of graft fixation on the femoral side (-Fig. 15).

\section{Harvest Site Complications}

Harvest site complications occur more commonly with BPTB versus hamstring autografts. ${ }^{36}$ ВРТВ donor harvest site complications include patellofemoral pain, kneeling pain, rupture or avulsion of the remaining patellar tendon, patellar fracture, patellar tendinosis, and quadriceps weakness. It was reported that abnormal signal within the patellar tendon after 12 months is indicative of patellar tendinosis., 92

It should be noted that extensor mechanism dysfunction and anterior knee pain after ACL reconstruction is common and may not be secondary to BPTB graft harvesting. The incidence of anterior knee pain was reported to be as high as $47 \%$ and is multifactorial. ${ }^{4}$ Preoperative anterior knee pain, preexisting patellofemoral cartilage damage, quadriceps weakness, concomitant flexion contracture, and overuse of 


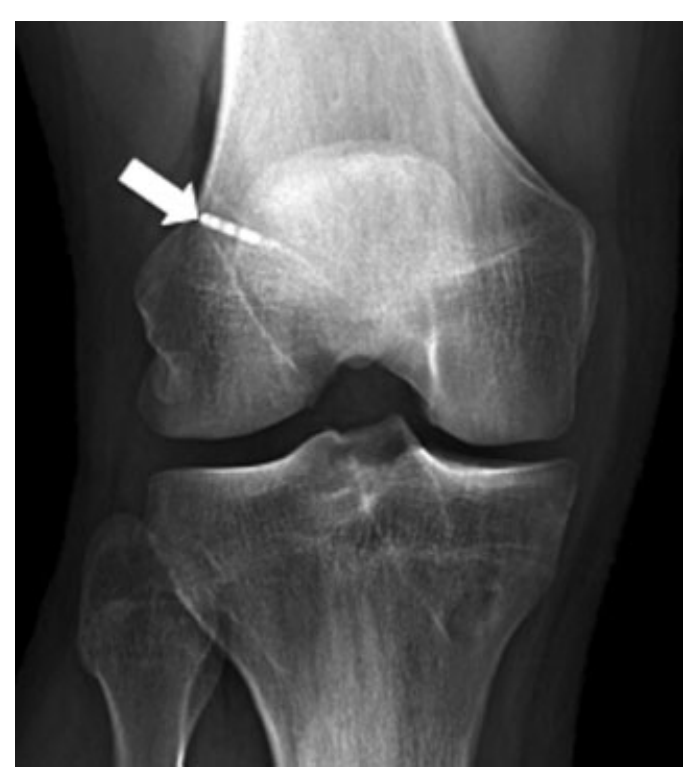

Fig. 15 Anteroposterior radiograph of a 41-year-old man demonstrates displacement of the EndoButton into the femoral tunnel (arrow).

open chain exercises all increase the risk of postoperative anterior knee pain. Another potential complication is patellar tendon contracture that may be associated with loss of motion (infrapatellar contraction syndrome). ${ }^{63}$ This may be the result of strict postoperative immobilization, lack of quadriceps activation postoperatively, and infrapatellar fat pad inflammation as a result of an exaggerated inflammatory response to surgery. Patellar tendon contracture will lead to increased patellofemoral contact forces and increased anterior translation of the tibia at terminal extension. Proper surgical technique and postoperative rehabilitation protocols encouraging early range of motion can prevent many of the causes of extensor mechanism dysfunction.

An additional complication associated with BPTB autografts is patellar fracture, a rare complication with an incidence of $\sim 1 \%$ after BPTB autograft ACL reconstruction. ${ }^{64,65}$ These fractures are typically the result of indirect trauma with the knee in flexion. Reports showed that harvesting BPTB grafts weakens the patella and increases the maximum stress on the bone. ${ }^{64}$ The origin of these fractures is multifactorial, depending on the size of the bone defect, the technique of bone plug harvest, the aggressiveness of rehabilitation, and the type of trauma that causes the fracture. With regard to the size of the bone plug, many authors state that the plug should not exceed 9 to $10 \mathrm{~mm}$ in width and depth. ${ }^{64}$ Nondisplaced fractures are treated nonoperatively, whereas displaced fractures usually require open reduction and internal fixation.

Hamstring tendon autografts are associated with less donor site morbidity and less extensor mechanism dysfunction. Potential complications related to hamstring autograft harvesting include injury to the medial collateral ligament and saphenous nerve and its branches during harvesting and postoperative hamstring weakness. ${ }^{11,66}$

\section{Osteoarthritis}

As mentioned previously, one of the goals of ACL reconstruction is to restore knee stability to reduce the rate of progression of cartilage and meniscal injury and resulting premature arthritis. It was shown, however, that ACL reconstruction may in fact be a risk factor for osteoarthritis as a long-term complication, 2 to 15 years after surgery. ${ }^{34}$ This is presumably due to alteration of normal biomechanics leading to compression of articular cartilage. This may affect both femorotibial and patellofemoral compartments. Risk factors for developing osteoarthritis after ACL reconstruction include meniscal injury, an interval $>6$ months between injury and surgery, and age $>25$ years at the time of surgery. ${ }^{67}$

\section{Conclusions}

ACL reconstruction is one of the most commonly performed orthopaedic procedures with several surgical options available and significant recent advances in technique. Thus the radiologist must be familiar and up to date with these different procedures and the complications that may occur. In this article, we reviewed the various surgical options available for ACL reconstruction, the normal postoperative imaging appearance after ACL reconstruction, and the potential complications that may occur.

\section{References}

1 Facchetti L, Schwaiger BJ, Gersing AS, et al; UCSF-P50-ACL Consortium; AF-ACL Consortium. Cyclops lesions detected by MRI are frequent findings after ACL surgical reconstruction but do not impact clinical outcome over 2 years. Eur Radiol 2017;27(08):3499-3508

2 Evans NA, Jackson DW. Arthroscopic management of the anterior cruciate ligament-deficient knee. In: McGinty JB, Burkhart SS, Jackson RW, Johnson DH, Richmond JC, eds. Operative Arthroscopy. Philadelphia, PA: Lippincott Williams \& Wilkins; 2003:347-365

3 Sanders TL, Maradit Kremers H, Bryan AJ, et al. Incidence of anterior cruciate ligament tears and reconstruction: a 21-year populationbased study. Am J Sports Med 2016;44(06):1502-1507

4 Allen CR, Giffin JR, Harner CD. Revision anterior cruciate ligament reconstruction. Orthop Clin North Am 2003;34(01):79-98

5 Reinhardt KR, Hetsroni I, Marx RG. Graft selection for anterior cruciate ligament reconstruction: a level I systematic review comparing failure rates and functional outcomes. Orthop Clin North Am 2010;41(02):249-262

6 Crawford SN, Waterman BR, Lubowitz JH. Long-term failure of anterior cruciate ligament reconstruction. Arthroscopy 2013;29 (09):1566-1571

7 Freedman KB, D’Amato MJ, Nedeff DD, Kaz A, Bach BR Jr. Arthroscopic anterior cruciate ligament reconstruction: a metaanalysis comparing patellar tendon and hamstring tendon autografts. Am J Sports Med 2003;31(01):2-11

8 Samuelsen BT, Webster KE, Johnson NR, Hewett TE, Krych AJ. Hamstring autograft versus patellar tendon autograft for ACL reconstruction: is there a difference in graft failure rate? A meta-analysis of 47,613 patients. Clin Orthop Relat Res 2017;475(10):2459-2468

9 Giaconi JC, Allen CR, Steinbach LS. Anterior cruciate ligament graft reconstruction: clinical, technical, and imaging overview. Top Magn Reson Imaging 2009;20(03):129-150

10 Williams RJ III, Hyman J, Petrigliano F, Rozental T, Wickiewicz TL. Anterior cruciate ligament reconstruction with a four-strand hamstring tendon autograft. J Bone Joint Surg Am 2004;86-A(02): 225-232 
11 Charalambous CP, Kwaees TA. Anatomical considerations in hamstring tendon harvesting for anterior cruciate ligament reconstruction. Muscles Ligaments Tendons J 2013;2(04):253-257

12 Tuman JM, Diduch DR, Rubino LJ, Baumfeld JA, Nguyen HS, Hart JM. Predictors for hamstring graft diameter in anterior cruciate ligament reconstruction. Am J Sports Med 2007;35(11): 1945-1949

13 Marrale J, Morrissey MC, Haddad FS. A literature review of autograft and allograft anterior cruciate ligament reconstruction. Knee Surg Sports Traumatol Arthrosc 2007;15(06):690-704

14 Konrath JM, Vertullo CJ, Kennedy BA, Bush HS, Barrett RS, Lloyd DG. Morphologic characteristics and strength of the hamstring muscles remain altered at 2 years after use of a hamstring tendon graft in anterior cruciate ligament reconstruction. Am J Sports Med 2016;44(10):2589-2598

15 Bryant AL, Creaby MW, Newton RU, Steele JR. Dynamic restraint capacity of the hamstring muscles has important functional implications after anterior cruciate ligament injury and anterior cruciate ligament reconstruction. Arch Phys Med Rehabil 2008;89 (12):2324-2331

16 Qu J, Thoreson AR, An KN, Amadio PC, Zhao C. What is the best candidate allograft for ACL reconstruction? An in vitro mechanical and histologic study in a canine model. J Biomech 2015;48 (10):1811-1816

17 Kaeding CC, Aros B, Pedroza A, et al. Allograft versus autograft anterior cruciate ligament reconstruction: predictors of failure from a MOON prospective longitudinal cohort. Sports Health 2011;3(01):73-81

18 Stucken C, Garras DN, Shaner JL, Cohen SB. Infections in anterior cruciate ligament reconstruction. Sports Health 2013;5(06): 553-557

19 Legnani C, Ventura A, Terzaghi C, Borgo E, Albisetti W. Anterior cruciate ligament reconstruction with synthetic grafts. A review of literature. Int Orthop 2010;34(04):465-471

20 Liu ZT, Zhang XL, Jiang Y, Zeng BF. Four-strand hamstring tendon autograft versus LARS artificial ligament for anterior cruciate ligament reconstruction. Int Orthop 2010;34(01):45-49

21 Lavoie P, Fletcher J, Duval N. Patient satisfaction needs as related to knee stability and objective findings after ACL reconstruction using the LARS artificial ligament. Knee 2000;7(03):157-163

22 Proffen BL, Sieker JT, Murray MM. Bio-enhanced repair of the anterior cruciate ligament. Arthroscopy 2015;31(05):990-997

23 Vavken P, Sadoghi P, Murray MM. The effect of platelet concentrates on graft maturation and graft-bone interface healing in anterior cruciate ligament reconstruction in human patients: a systematic review of controlled trials. Arthroscopy 2011;27(11): 1573-1583

24 Murray MM, Spindler KP, Abreu E, et al. Collagen-platelet rich plasma hydrogel enhances primary repair of the porcine anterior cruciate ligament. J Orthop Res 2007;25(01):81-91

25 Hirzinger C, Tauber M, Korntner S, et al. ACL injuries and stem cell therapy. Arch Orthop Trauma Surg 2014;134(11):1573-1578

26 Silva A, Sampaio R, Fernandes R, Pinto E. Is there a role for adult non-cultivated bone marrow stem cells in ACL reconstruction? Knee Surg Sports Traumatol Arthrosc 2014;22(01):66-71

27 Dienst M, Burks RT, Greis PE. Anatomy and biomechanics of the anterior cruciate ligament. Orthop Clin North Am 2002;33(04): 605-620, v

28 Breland R, Metzler A, Johnson DL. Indications for 2-incision anterior cruciate ligament surgery. Orthopedics 2013;36(09): 708-711

29 Rayan F, Nanjayan SK, Quah C, Ramoutar D, Konan S, Haddad FS. Review of evolution of tunnel position in anterior cruciate ligament reconstruction. World J Orthop 2015;6(02):252-262

30 Meredick RB, Vance KJ, Appleby D, Lubowitz JH. Outcome of single-bundle versus double-bundle reconstruction of the anterior cruciate ligament: a meta-analysis. Am J Sports Med 2008;36 (07):1414-1421
31 Yang J, Fu S, Song Y. A prospective randomized controlled trial to evaluate clinico-radiological outcome of arthroscopic single bundle versus double bundle anterior cruciate ligament reconstruction. Int J Clin Exp Med 2017;10(01):1087-1093

32 Casagranda BU, Maxwell NJ, Kavanagh EC, Towers JD, Shen W, Fu FH. Normal appearance and complications of double-bundle and selective-bundle anterior cruciate ligament reconstructions using optimal MRI techniques. AJR Am J Roentgenol 2009;192(05): 1407-1415

33 Lee YS, Nam SW, Sim JA, Lee BK. Comparison of anatomic ACL reconstruction between selective bundle reconstruction and double-bundle reconstruction. Knee Surg Sports Traumatol Arthrosc 2014;22(11):2803-2810

34 Zappia M, Capasso R, Berritto D, et al. Anterior cruciate ligament reconstruction: MR imaging findings. Musculoskelet Surg 2017; 101(Suppl 1):23-35

35 Recht MP, Kramer J. MR imaging of the postoperative knee: a pictorial essay. Radiographics 2002;22(04):765-774

36 Meyers $A B$, Haims AH, Menn $K$, Moukaddam $H$. Imaging of anterior cruciate ligament repair and its complications. AJR Am J Roentgenol 2010;194(02):476-484

37 Saupe N, White LM, Chiavaras MM, et al. Anterior cruciate ligament reconstruction grafts: MR imaging features at longterm follow-up-correlation with functional and clinical evaluation. Radiology 2008;249(02):581-590

38 Malinin TI, Levitt RL, Bashore C, Temple HT, Mnaymneh W. A study of retrieved allografts used to replace anterior cruciate ligaments. Arthroscopy 2002;18(02):163-170

39 Kim M, Choi YS, Kim H, Choi NH. Postoperative evaluation after anterior cruciate ligament reconstruction: measurements and abnormalities on radiographic and CT imaging. Korean J Radiol 2016;17(06):919-930

40 Denti M, Lo Vetere D, Bait C, Schönhuber H, Melegati G, Volpi P. Revision anterior cruciate ligament reconstruction: causes of failure, surgical technique, and clinical results. Am J Sports Med 2008;36(10):1896-1902

41 Bencardino JT, Beltran J, Feldman MI, Rose DJ. MR imaging of complications of anterior cruciate ligament graft reconstruction. Radiographics 2009;29(07):2115-2126

42 Bernard M, Hertel P, Hornung H, Cierpinski T. Femoral insertion of the ACL. Radiographic quadrant method. Am J Knee Surg 1997;10 (01):14-21; discussion 21-22

43 Dhawan A, Gallo RA, Lynch SA. Anatomic tunnel placement in anterior cruciate ligament reconstruction. J Am Acad Orthop Surg 2016;24(07):443-454

44 Rizer M, Foremny GB, Rush A III, et al. Anterior cruciate ligament reconstruction tunnel size: causes of tunnel enlargement and implications for single versus two-stage revision reconstruction. Skeletal Radiol 2017;46(02):161-169

45 Horton LK, Jacobson JA, Lin J, Hayes CW. MR imaging of anterior cruciate ligament reconstruction graft. AJR Am J Roentgenol 2000;175(04):1091-1097

46 Rispoli DM, Sanders TG, Miller MD, Morrison WB. Magnetic resonance imaging at different time periods following hamstring harvest for anterior cruciate ligament reconstruction. Arthroscopy 2001;17(01):2-8

47 Bach BR Jr. Revision anterior cruciate ligament surgery. Arthroscopy 2003;19(10, Suppl 1):14-29

48 White LM, Kramer J, Recht MP. MR imaging evaluation of the postoperative knee: ligaments, menisci, and articular cartilage. Skeletal Radiol 2005;34(08):431-452

49 Naraghi AM, Gupta S, Jacks LM, Essue J, Marks P, White LM. Anterior cruciate ligament reconstruction: MR imaging signs of anterior knee laxity in the presence of an intact graft. Radiology 2012;263(03):802-810

50 Gohil S, Falconer TM, Breidahl W, Annear PO. Serial MRI and clinical assessment of cyclops lesions. Knee Surg Sports Traumatol Arthrosc 2014;22(05):1090-1096 
51 Muellner T, Kdolsky R, Grossschmidt K, Schabus R, Kwasny O, Plenk H Jr. Cyclops and cyclopoid formation after anterior cruciate ligament reconstruction: clinical and histomorphological differences. Knee Surg Sports Traumatol Arthrosc 1999;7(05):284-289

52 Simpfendorfer C, Miniaci A, Subhas N, Winalski CS, Ilaslan H. Pseudocyclops: two cases of ACL graft partial tears mimicking cyclops lesions on MRI. Skeletal Radiol 2015;44(08):1169-1173

53 Ekhtiari S, Horner NS, de Sa D, et al. Arthrofibrosis after ACL reconstruction is best treated in a step-wise approach with early recognition and intervention: a systematic review. Knee Surg Sports Traumatol Arthrosc 2017;25(12):3929-3937

54 Orsi AD, Canavan PK, Vaziri A, Goebel R, Kapasi OA, NayebHashemi $H$. The effects of graft size and insertion site location during anterior cruciate ligament reconstruction on intercondylar notch impingement. Knee 2017;24(03):525-535

55 Seeger LL, Sako E, Motamedi K, Levine BD, Hame SL. Intra-articular calcifications following arthroscopic ACL reconstruction: prevalence and possible significance. Skeletal Radiol 2014;43(02): 209-212

56 Ghazikhanian V, Beltran J, Nikac V, Feldman M, Bencardino JT. Tibial tunnel and pretibial cysts following ACL graft reconstruction: MR imaging diagnosis. Skeletal Radiol 2012;41(11): 1375-1379

57 Weber AE, Delos D, Oltean HN, et al. Tibial and femoral tunnel changes after ACL reconstruction: a prospective 2-year longitudinal MRI study. Am J Sports Med 2015;43(05):1147-1156

58 Emond CE, Woelber EB, Kurd SK, Ciccotti MG, Cohen SB. A comparison of the results of anterior cruciate ligament reconstruction using bioabsorbable versus metal interference screws: a meta-analysis. J Bone Joint Surg Am 2011;93(06):572-580
59 Pereira H, Correlo VM, Silva-Correia J, Oliveira JM, Reis RL, Espregueira-Mendes J. Migration of "bioabsorbable" screws in ACL repair. How much do we know? A systematic review. Knee Surg Sports Traumatol Arthrosc 2013;21(04):986-994

60 Studler U, White LM, Naraghi AM, et al. Anterior cruciate ligament reconstruction by using bioabsorbable femoral cross pins: MR imaging findings at follow-up and comparison with clinical findings. Radiology 2010;255(01):108-116

61 Mae T, Kuroda S, Matsumoto N, et al. Migration of EndoButton after anatomic double-bundle anterior cruciate ligament reconstruction. Arthroscopy 2011;27(11):1528-1535

62 Tuite MJ, De Smet AA. MR of the postoperative knee. Top Magn Reson Imaging 1996;8(01):2-14

63 Paulos LE, Wnorowski DC, Greenwald AE. Infrapatellar contracture syndrome. Diagnosis, treatment, and long-term followup. Am J Sports Med 1994;22(04):440-449

64 Stein DA, Hunt SA, Rosen JE, Sherman OH. The incidence and outcome of patella fractures after anterior cruciate ligament reconstruction. Arthroscopy 2002;18(06):578-583

65 Tay GH, Warrier SK, Marquis G. Indirect patella fractures following ACL reconstruction: a review. Acta Orthop 2006;77(03): 494-500

66 Hardy A, Casabianca L, Andrieu K, Baverel L, Noailles T; Junior French Arthroscopy Society. Complications following harvesting of patellar tendon or hamstring tendon grafts for anterior cruciate ligament reconstruction: systematic review of literature. Orthop Traumatol Surg Res 2017;103(8S):S245-S248

67 Seon JK, Song EK, Park SJ. Osteoarthritis after anterior cruciate ligament reconstruction using a bone-patellar tendon-bone autograft. Am J Sports Med 2006;29:450-456 